\title{
Dialogue sur l'utopie, le féminisme et autres sujets connexes
}

\author{
Élisabeth Vonarburg interviewée par Sylvie Bérard
}

\begin{abstract}
Élisabeth Vonarburg, award-winning québécoise writer of such feminist science-fiction/fantasy novels as Le Silence de la Cité and Chroniques du Pays des Mères (published in translation as The Silent City and The Maerlande Chronicles), is here interviewed by Sylvie Bérard. They discuss such questions as what qualifies a utopian representation as feminist? what are the epistemological and political stakes of the transposition of sexual differentiation to distant times and places? how and why is a writer led to the genres of science fiction and/or utopia? can feminist fiction sustain a plurality of points of view?
\end{abstract}

Laissez-moi d'abord mettre en contexte cette interview. Élisabeth Vonarburg publie de la science-fiction depuis près de vingt ans et elle en lit depuis encore plus longtemps. Outre un nombre respectable de nouvelles et d'articles, et même d'une thèse de doctorat sur la question, on lui doit des romans tels que Le Silence de la cité et Les Voyageurs malgré eux, et, plus récemment, un cycle de cinq romans intitulé «Tyranaël». Parmi toute cette production figure un roman qu'on pourrait considérer comme étant son utopie féministe - même si l'auteure se refuse à cette étiquette, un roman singulier dans la production francophone mais qui se fait l'écho et se prolonge par toute une série d'utopies ou de dystopies féministes anglo-saxonnes: The Female Man de Joanna Russ, Walk to the End of the World et The Furies de Suzy McKee Charnas, The Gate to the Women's Country de Sheri S. Tepper etc. Ce roman, Chroniques du Pays des Mères, fut très bien reçu par la critique et traduit en anglais (Canada: The Maerlande Chronicles; États-Unis: as In the Mother's Land) et, récemment, en allemand. Avec cette chronique des temps futurs où les femmes dominent (démographiquement du moins), Élisabeth Vonarburg a pénétré le marché québécois, canadien et nord-américain, tant du côté de la sciencefiction que du côté de la littérature générale en obtenant plusieurs prix littéraires (Aurora Award for Best Science Fiction and Fantasy Book in French, Grand Prix Logidec de la SF québécoise, Prix Boréal du meilleur roman, Philip K. Dick Special Jury Award). Il a été recensé dans plusieurs 
périodiques d'importance, dont les magazines de science-fiction SFRA Review et The New York Review of Science Fiction. En général, la plupart des critiques ont souligné la qualité du style et l'acuité de la vision. féministe et de la féminisation du monde, tant dans la version originale que dans la traduction. Ce succès était en fait la récompense de plusieurs années de travail et de réflexion. Élisabeth Vonarburg, en effet, avait préparé ce livre pendant longtemps. Déjà, en 1980, elle avait cette histoire en tête lorsqu'elle avait commencé la rédaction de son roman précédent.

Toutefois, ce qui aurait dû demeurer un bref épisode au sein de ce qu'elle prévoyait être une trilogie du Pays des Mères s'est transformé en un roman autonome qu'elle intitula Le Silence de la cité. Ses lecteurs et lectrices devraient attendre dix longues années avant de lire Chroniques $d u$ Pays des Mères. L'action de ce roman est située dans un futur lointain par rapport au Silence de la cité, à une époque où l'on commence à découvrir que toute une technologie s'est perdue à travers les âges, et où, en raison d'un mystérieux fléau, plus de quatre-vingt-dix pour cent de la population est de sexe féminin. Lisbeï, le personnage principal, va tenter de démystifier le passé tout en se réconciliant avec sa propre destinée et sa propre identité. La forme en soi, avec sa triple temporalité et ses voix multiples, tente de recréer le dialogue qui s'établit entre le personage et elle-même. Ce système social fictif où règnent les femmes est également soutenu par tout un système grammatical selon lequel, contrairement à l'usage du français, le féminin l'emporte sur le masculin. Bref, tout cela était de nature à $m$ 'inciter à poser à l'auteure quelques questions dans le contexte de ce numéro sur les utopies féministes. Voici donc la version remaniée d'un petit dialogue plutôt spontané que nous avons tenu par courrier électronique au printemps 1999.

SB: Une première question qu'on peut se poser touche les raisons pour lesquelles on (et ce n'est pas toujours les auteures qui le font) qualifie telle ou telle oeuvre d'utopie féministe. On appose parfois cette étiquette à des romans construisant un monde fictionnel où les hommes ont été décimés par un act of God. Non mais, quelle belle vision du féminisme ! Qu'en penses-tu?

Pourquoi, au fait? S'ils étaient défectueux et si Elli avait tout crée, pourquoi avait-Elli fait des mosta ratées? La question n'avait cependant pas été posée en ces termes. «Pourquoi il n'y a pas beaucoup de garçons? avait un jour demandé Turri, ou Rubbio, ou Garrec. - C'est une puni- 
tion envoyée par Elli», répondit la gardienne, sans doute la vieille Tessa, après une petite hésitation cependant. (Chroniques du Pays des Mères, p. 40)

EV: A relativiser: qui «qualifie automatiquement»? Des féministes ou des non-féministes (femmes ou hommes, bien sûr)? Si des féministes, quelle variété de féministes ? Ne pas oublier que ce motif-là est aujourd'hui considéré comme daté, tout comme les romans dits séparatistes. Il faut replacer dans l'époque où ces romans-là ont été produits. Pourquoi ne dit-on pas aussi dystopies féministes, au fait ? et en tout cas le bouquin de Charles Maine (Alph) qui a suscité en moi la crise profonde d'urticaire donnant lieu à Chroniques, et au Silence par rebonds, n'était vraiment pas un roman féministe, ni une utopie féministe, bien que les hommes y eussent été décimés. Ceci dit, ce n'est pas forcément une «belle vision du féminisme», mais on (les femmes) n'est pas là pour se conférer la sainteté ; utopie ou en fin de compte dystopie, c'est sûrement une vision bien claire et bien sincère, à l'époque et encore maintenant, des sentiments profonds de beaucoup de femmes à l'égard des hommes dans nos sociétés patriarcales (dont auxquelles on sait bien que etc.). Les textes où les femmes ont disparu sont bien plus rares, tiens donc, curieux (je n'en ai qu'un en mémoire, écrit par un homme, une nouvelle: Jean-Claude Dunyach, «En attendant les porteurs d'enfants»), et suffisamment rares même que ça vaudrait le coup d'aller y voir. Et entre les deux bien entendu, Philip Wylie, The Disappearance où chacun, les hommes, les femmes, disparaît aux yeux de l'autre.

SB: Mais quels sont donc les attraits féministes de l'utopie par rapport à d'autres formes? Pourquoi construire une utopie explicite, si l'on peut dire, alors que, peut-être, tout roman féministe qui ne se contente pas de dénoncer mais de montrer comment ce serait si c'était mieux est en fait une utopie ? Crois-tu que l'utopie est particulièrement bien placée pour adopter un point de vue féministe? Selon toi, l'utopie a-t-elle une portée politique, et si oui quelle est cette portée?

C'est étrange ; toutes ces années que j'ai passées à sauter d'univers en univers à la recherche de ce que je ne reconnaîtrais pas, et jamais je ne me suis demandée comment je le reconnaîtrais, cet autre arbre-à-univers. La différence absolue, comment la reconnaître? Peut-on même la percevoir ? Lorsque j'y pensais, je me disais que ce serait une sorte d'illumination, que je saurais. Je n'avais pas songé que ce devait être une illumination 
sans retour : qui vous change irrémédiablement, qui vous arrache à vousmême. Comment connaître l'autre absolu, si on emporte partout son propre reflet? On ne peut que reconnaître. Il faut être l'autre, ne plus être soimême... Impossible de ne pas penser «cesser d'être». («Le jeu des coquilles de nautilus», p. 288)

EV: Oui - à condition que ce soit l'utopie ambiguë de Le Guin - ce qu'elle a d'ailleurs toujours été, chez les féministes et consort(e)s depuis les années 60 : à ce que je sache, aucun des livres de SF écrits par des femmes examinant "comment ce serait si c'était mieux" - ou autrement - ne se sont présentés comme des utopies au sens classique (et l'Euguélionne de Bersianik non plus, non ? zut! toujours pas fait mes devoirs et lu Les Guérillères de Wittig...). C'était/c'est de la SF, des sociétés en évolution, imparfaites etc. Le roman de Sargent The Shore of Women présente diégétiquement une utopie classique dominée par des femmes, mais justement pour la critiquer comme dystopie... Et à ce que je sache, The Gate to the Women's Country de Tepper n'est pas, dans l'esprit même de son auteure, une utopie. Dans les plus récentes sociétés dominées par les femmes, disons, et tout relativement, ni Cherryh ni Arnasson ne prétendent utopiser.

Je ne connais pas assez les textes des utopies féministes de la fin du 19e et du début du 20e, malheureusement, pour en parler plus doctement. Était-ce des utopies bon teint (i.e. non ambiguës) ? Beaucoup en tout cas, semblent être très proches de la SFS. Ce que je sais/crois, en tout cas, c'est qu'utopie et science-fiction ont partie liée historiquement, et que féminisme, science-fiction et utopie aussi : (a) Les écrivaines feministes qui veulent par définition sortir réalistement du statu quo doivent fictionnellement plonger d'une façon ou d'une autre dans le futur et donc dans la SF (ou le devraient bien !) De plus, [ne] doit-on [pas aussi] considérer les stratégies utopisantes de la Fantasy? (b) Et si elles ne veulent pas répéter la même antienne constatrice déprimante-revendicatrice-stridente, elles doivent imaginer comment c'est... au moins différent; de différent à mieux, le pas est mince à franchir, et les pressions de la SF (comme genre) jouent souvent dans ce sens d'où l'effet utopique à la réception, du moins chez certains mal informés [rire].

SB: Alors je pose la question inverse: est-ce qu'un roman, développe-t-il une utopie, peut-être considéré comme un discours féministe (avec insistance sur le singulier et sur la nature de la prise de parole). Pour faire un roman, est-il nécessaire d'adopter une pluralité de points de vue? 
EV: Euh, si l'ellipse entre les deux questions concerne le fait que la fiction utopiste n'a pas de pluralités de point de vue : eh bien... elle en a au moins deux : ou bien celui du Visiteur et celui du Prêcheưr, ou bien celle du lecteur (visiteur implicite) et celle du Prêcheur. Ça suffit à faire un roman, non ? Et on peut faire un roman avec un seul point de vue (y a-til deux points de vue dans L'étranger de Camus, ou La nausée de Sartre, par exemple?

SB: Une autre chose me chicote. Ça s'éloigne un peu de ton corpus à proprement parler et un peu de l'utopie, mais il faut compter aussi tes traductions - je songe à Leviathan's Deep de Carr. Par quel implicite une auteure d'une fiction féministe décide-t-elle que cette moitié-là de sa race extra-terrestre est masculine et l'autre moitié féminine et, partant, construit une fiction à la limite de l'utopie féministe. Autrement dit, est-ce qu'en voulant déplacer les problématiques humaines pour les mettre à distance on ne retombe pas en plein dans le piège des catégorisations sexistes en apposant à des créatures non humaines certains traits humains qui les font pencher du côté masculin ou du côté féminin (au détriments de certaines autres caractéristiques, peut-être, dont pourrait découler le parti pris identitaire inverse) ?

Ne pas porter ma responsabilité - ma culpabilité. Encore maintenant, ou sinon pourquoi aurais-je passé les sans doute dernières semaines de ma vie à rassembler tous ces Rêves, à échafauder encore ces histoire Rêvées avec les autres, où j'étais l'autre, l'étranger ou l'étrangère? Maintenant encore, après tout ce temps, je n'arrive pas à vraiment admettre mon innocence - l'absurdité de mon destin. Et même si c'était la volonté de la Mer, ses desseins seraient tout aussi insondables que le hasard. (Remplacer une énigme par un mystère), disait Shandaar. (Les rêves de la Mer, $p$. 362)

EV: Mmmm. Pour Carr, il me semble que tu considères le présent problème comme résolu, c'est-à-dire que son texte est une utopie féministe... Mais bon. Je sais pas trop(e). Je n'ai pas traduit par exemple Arnasson, qui me semble l'auteure-type à qui poser cette question. Ou Cherryh (dieu sait !). Et je n'ai pas traduit non plus le Bradley (The Planet Wreckers) réponse de la bergère Bradley à la bergère Le Guin, où l'extraterrestre hermaphrodite fait l'amour en mode femelle avec l'humain mâle et en a même un enfant en restant il (wow, mind blowing!). Dans mes écritures, où il n'y a pratiquement pas d'extraterrestres de toute 
manière (deux ou trois dans des nouvelles, et les habitants de la planète $X$ où règne 1'Entité des Voyageurs lesquels ET sont de toute façon des amorces de métamorphes), ce qui m'intéresse, justement, ce sont des ET qui ne sont ni mâles ni femelles ou les deux. Je te renvoie au texticule paru au temps jadis dans La Nouvelle Barre du Jour, où toute l'histoire tourne autour du sexe des (anges) ET, à sa façon. J'ai une nouvelle avec mâles et femelles Et («Les yeux ouverts»), mais issue malgré tout de l'arbitraire d'un exercice d'atelier, et pas forcément representative... Et bien sûr, tous le toutim tyranaëlien, où j'ai joué très délibérément (en évoluant beaucoup au cours des années, et en m'éloignant le plus possible des origines utopiques-classiques de mes ET - pas féministe du tout, l'origine - mais avec un succès mitigé) avec les machins masculins-féminins (me rapprochant donc d'une... utopisation féministe ? La société rani est bien plus vivable de ce point de vue que la nôtre et donc...)... L'a priori de base étant évidemment que c'est malgré tout une race bisexuée, ce qui me semble déjà pas mal contraint/contraignant au départ!

Maintenant ( pourquoi ) ? euh... Parce que je suis une être sexuée, et parce que je suis potentiellement ambisexuelle. Que sais-je ?!! [rire] Mon utopie à moi, mon mythe fondateur personnel ne comprend pas vingtcinq sexes, et n'est pas monosexe, malgré certaines tentations ponctuelles, voilà. Je fais partie des timides-modérées-vanille. On pourrait aussi se demander pourquoi diable toutes les utopies, puisque ce sont des utopies, hein, enfin, ne sont pas féministes ! On nage en plein syllogisme, là.

SB: Du côté de ton écriture, maintenant, vois-tu une différence dans le processus qui te mène à l'utopie par rapport à celui qui te fait élaborer un texte de SF (comparons par exemple l'écriture du Silence aux Chroniques) ? Sont-ce simplement des différences qui, de toute manière, existent dans l'écriture de tout roman par rapport à un autre?

Élisa considère les traces de ses dents dans la peau luisante de la pomme: deux arcs de cercle symétriques. Elle mord dans l'autre sens: le cercle est complet à présent. Quand elle mordre pour la troisième fois dans la pomme, il y aura un trou. et ainsi de suite, et à chaque fois l'aspect de la pomme se modifiera. Mais ce sera toujours une pomme. C'est son sens qui changera, le sens que je lui donnerai. (Le silence de la cité, p. 319)

EV: Chroniques n'est pas une utopie, ni dans mon intention (je l'ai justement entrepris contre une (utopie féministe vue par un mec, rappel) ni 
dans sa réalisation, ni dans les multiples lectures qui en ont été faites jusqu'à ce jour, me semble-t-il (y compris la bouchardienne [rire]). Mais c'est un roman féministe. Avec dialogisme (coucou, Bakhtine). Et Le Silence non plus, puisqu'y est critiquée la position initiale des utopistes classiques et mâles tels que Paul, qui ne se définit jamais comme tel d'ailleurs, au contraire! Mais la problématique est présente dans sa pratique, bien sûr, laquelle expose la dystopie totalitaire fondamentale à toute utopie classique, tout comme au reste l'envers de la dystopie masculine, soit la position séparatiste-totalitaire aussi des Femmes Libres, est également décrit dans le roman. Et me voilà coincée avec comme voix de l'auteure dans Le Silence un homme (Manilo, qui passe pour le défenseur du statu quo, alors que c'est un réformiste évolutionniste... comme moi) et une femme aliénée, Élisa. Pfff...

SB: Mais alors, que faire, en tant qu'auteure de science-fiction intéressée par l'utopie (tu vois, je suis maintenant prudente) avec toutes ces questions d'utopie féministe, de cohabitation des points de vue, de la dénonciation des éléments dystopiques, des propositions utopiques, etc.?

Les résultats des croisements ponctuels de l'entité, au cours des siècles, avec la vie indigène de la planète? Ou imités des formes de vie dont se souvenaient les Voyageurs? Un multitude d'hypothèses se pressaient dans l'esprit de Catherine, mais elle les écarts résolument. Il serait toujours temps pour les spéculations, les questions et les réponses. Elle préférait être là, maintenant, dans cette forêt qui sentait la mousse et les champignons au travers de toutes ses autres odeurs étrangères, excitantes. (Les voyageurs malgré eux, $p .420$ )

EV: Moi ce qu'il m'intéresse d'écrire, ce sont les questions, tout ce qui rend les questions possibles (nécessaires), et tous les tortillements des réponses dans le temps et en grandeur réelle, enfin réelle dans une fiction ayant partie (honteusement) liée avec le réalisme par le biais du faut-que-les-lecteurices-embarquent. À ces fins, anything goes. Voilà tout ce que je peux te dire de songé sur mon écriture de ma para-néohypothético-utopie, pour le moment. «Le processus qui me mène à l'utopie par rapport à celui qui me fait élaborer un texte de SF»... bien, je n'écris que de la SF ! Le chemin qui me fait éviter l'utopie m'a menée à la $\mathrm{SF}$, alors ! [rire] J'ai été naturellement portée vers la nature quand même (j'aime bien le «quand même») science-fictionnelle (moins à thèse que l'utopie, peut-être ?) de Chroniques du Pays des Mères, par exemple. Le «à 
thèse» me fait toujours suer, comme lectrice et comme auteure, et comme cible des réactions et questions (niaises alors pour moi) des réceptrices. Un des aspects qui m'agace dans la SF, c'est le pédagogisme. Une des critiques les plus urticantes qu'on puisse me faire (qu'on m'a faites), pour Le Silence c'est d'y voir du philosophisme, du moralisme, des thèses ; pour Chroniques aussi bien. Une des critiques les plus agréables qu'on m'ait faites, c'est (ah-ah-ah) «l'absence louable» de pédagogisme, moralisme et cie dans ces bouquins. Je (trouve que je) m'en suis sûrement moins bien dépatouillée cependant (au plan de l'écriture) dans Le Silence que dans Chroniques... Mon principal problème d'écriture (je n'ose le dire à mes ami(e)s littératurantes), que j'ai tendance à considérer comme le problème d'écriture spécifique à la SF (et sans doute au thriller, tiens... ou à certains types de - bonne - fantasy où on crée un monde cohérent), c'est (comment présenter sans effort et invisiblement même l'information nécessaire pour comprendre ce qui se passe?), c'est-à-dire pour comprendre pourquoi ces questions et pourquoi ces réponses-là de la part des personnages. Ça va des décors, paysages, objets, aux tenants et aboutissants socio-politico-biologico-historico-mythologico-alouetto-tique des univers en jeu, en passant par les personnages/projections entre autres de l'auteure.

SB: Est-ce que tu veux dire que le moment où la fiction devient intéressante, c'est là où elle dérape, que là où ne passe pas le contenu que l'auteure veut absolument nous transmettre, mais quelque chose d'autre? EV: Ah, le grand mythe du dérapage. Ou du moins faudrait-il le relativiser lui aussi. Toutes les auteures et leurs collègues masculins ne dérapent pas au même moment ni de la même façon. Je suis une écrivaine très organisée. Mes «dérapages» ont lieu pendant la période de remue-méninges, avant l'écriture elle-même, au moment où j'écris, certes, mais des kilomètres de notes, questions, réponses, essais, arguties, oui-mais-non-et-pourquoi-pas etc. - et ce parfois pendant des années. Ce que je dis dans une histoire, nouvelle et surtout roman, c'est au moins à $80 \%$ ce que je veux dire, parce que j'ai découvert auparavant, via un certain nombre de dérapages-découvertes dus aussi bien à la réflexion logique qu'aux plongées autres de l'émotion-besoin ou aux remontées du subconscient (ah la la, toute cette hiérarchie spatiale de l'esprit, mais la barbe! D'autant que mon expérience de la chose au moment «remueméninges» comme au moment «écriture» de la création, c'est une expérience holistique, et non séquentielle...). J'ai donc tendance à répondre ceci 
à ta question : pourquoi nos lapsus seraient-ils tous a priori plus intéressants que ce que nous voulons dire? Et, bien entendu, vice versa. Une parole est un tout. Un texte est un tout. Une histoire et un récit forment un tout. Une oeuvre, sapristi, est un tout ! Ce qui est «intéressant», c'est la dynamique des tensions qui s'y établissent. Comme entre l'utopie et la dystopie, ou entre le féminin et le masculin - avec toutes les possibilités entre les deux pour venir aménager ces tensions et les rendre humaines : vivables dans la diversité.

\section{Ouvrages cités}

Arnason, Eleanor. «The Lovers», dans Flying Cup and Saucer. Gender Explorations in Science Fiction \& Fantasy. Debbie Notkon and The Secret Feminist Cabal (Cambridge : Edgewood Press, 1998).

-. A Woman of the Iron People (New York: Morrow, 1991).

Bersianik, Louky. L'Euguélionne. Roman Triptyque (Montréal : La Presse, 1981).

Bradley, Marion Zimmer. The Worlds Wreckers (Boston : Gregg Press, 1979).

Carr, Jayge. Leviathan's Deep (New York: Playboy Press, 1980); traduction par Élisabeth Vonarburg, L'abîme de Léviathan (Paris : Albin-Michel, 1982).

Charnas, Suzy McKee. Walk to the End of the World (New York : Ballantine Books, 1974).

-. The Furies (New York: Tor, 1994).

-. The Conqueror's Child (New-York: Tor, 1999).

Dunyach, Jean-Claude. «En attendant les porteurs d'enfants», dans Science-fiction, 1 (hiver 1984): 57-74.

Maine, Charles. Alph (New York : Ballantine, 1972).

Russ, Joanna. The Female Man (New York : Bantam, 1975); traduction L'autre moitié de l'homme (Paris: Laffont, 1977).

Sargent, Pamela. The Shore of Women (New York : Bantam Spectra, 1987) ; traduction Le rivage des femmes (Paris: Le livre de poche, 1997).

Tepper, Sheri S. The Gate to Women's Country (New York: Double Day Foundation, 1988); traduction Un monde de femmes (Paris: J'ai lu, 1990). Vonarburg, Élisabeth. Le Silence de la cité (Paris : Denoël, 1981) ; traduction The Silent City (Victoria : Press Porcépic, 1988; republication (London: The Women's Press, 1990), New York : Bantam Spectra Special, 1992) ; traduction Die Schweigende Stadt (Germany: Wilhelm Heyne 
Verlag, 1997) ; édition révisée (Beauport: Alire, 1998).

—. Chroniques du Pays des Mères (Montréal: Québec/Amérique, 1992); traduction In the Mother's Land (New York: Bantam Spectra Special, 1992); republication The Maerlande Chronicles (Victoria: Beach Holme, 1992); traduction Die Maerlande Chroniken (Munich: Wilhelm Heyne Verlag, 1997) ; édition révisée (Beauport: Alire, [à paraître]).

—. Les Voyageurs malgré eux (Montréal: Québec/Amérique, 1994); traduction The Reluctant Voyagers (New York: Bantam Spectra, 1995 ; Edmonton : Tesseract Books, 1996).

—. Tyranaël I: Les Rêves de la Mer (Beauport: Alire, 1996).

—. Tyranaël II: Le Jeu de la Perfection (Beaupor: Alire, 1996).

—. Tyranaël III: Mon Frère l'ombre (Beauport: Alire, 1997).

—. Tyranaël IV: L'Autre Rivage (Beauport: Alire, 1997).

—. Tyranaël V: La Mer allée avec le soleil (Beauport: Alire, 1997).

-. «L'Or, l'encens et la myrrhe», dans La Nouvelle Barre du jour: Sciencefiction, 79-80 (juin 1979) : 119-131.

—. «Le jeu des coquilles de nautilus», dans Aurores Boréales II, Daniel Sernine dir. publ. (Longueuil: Éditions du Préambule, 1986) : 253-290

-. «Les yeux ouverts», dans Ailleurs et au Japon (Montréal:

Québec/Amérique, 1991).

Wittig, Monique. Les guérillères (Paris: Minuit,1969).

Wylie, Philip. The Disappearance (New York: Lightyear, 1993 [1951]). 